\title{
COMPENSATORY AND ADAPTIVE RESPONSES TO REAL-TIME FORMANT SHIFTS IN ADULTS AND CHILDREN
}

\author{
Hayo Terband ${ }^{1} \&$ Frits van Brenk $k^{1,2}$ \\ ${ }^{1}$ Utrecht Institute of Linguistics - OTS, Utrecht University \\ ${ }^{2}$ Division of Speech and Language Therapy, University of Strathclyde \\ h.r.terband@uu.nl; f.j.vanbrenk@uu.nl
}

\begin{abstract}
Auditory feedback plays an important role in speech motor learning. Previous studies investigating auditory feedback in speech development suggest that crucial steps are made in the development of auditory-motor integration around the age of 4 .

The present study investigated compensatory and adaptive responses to auditory perturbation in 4 to 9 year-old children compared to young adults (aged 19 - 29 years). Auditory feedback was perturbed by real-time shifting the first and second formant (F1 and F2) of the vowel /e:/ during the production of CVC words in a five-step paradigm (familiarization; baseline; ramp; hold; release).

Results showed that the children were able to compensate and adapt in a similar or larger degree compared to the young adults, even though the proportion of speakers displaying a consistent compensatory response was higher in the group of adults. In contrast to previous reports, results did not show differences in token-to-token variability between children and adults.
\end{abstract}

Keywords: speech; development; sensori-motor control; auditory feedback perturbation.

\section{INTRODUCTION}

The production of speech sounds is a sensori-motor accomplishment in which auditory feedback plays an important role, serving both as a teaching signal for the acquisition and adaptation of speech motor programs $[2,3,10,12]$ and as a guiding signal in the online control and correction of speech movements [10-12].

The auditory feedback perturbation paradigm comprises the creation of an apparent mismatch between the speech sound the speaker intended to produce and what he/she hears back. The acoustic signal is recorded during speech production and an acoustic cue is manipulated in real time and presented back to the speaker through headphones. Several studies demonstrated that the unexpected perturbation of auditory feedback during speech production elicits a compensatory response, usually in the opposite direction to maintain the intended auditory outcome [1, 4, 14]. Furthermore, sustained application of acoustic cue shifts causes the speech motor system to adapt to the perturbation and modify its speech motor programs. These perturbation studies have mainly focused on adult populations. As auditory feedback also plays an important role in speech motor learning, i.e. the acquisition of speech motor programs [2, 7-9], it is imperative to assess to what extent children are able to compensate for and adapt to auditory feedback perturbations throughout their developmental trajectory.

Thus far, only a few studies have utilized the auditory feedback perturbation paradigm in studies with children. Together, previous studies suggest that crucial steps are made in the development of auditory motor learning between the ages $4-9$. MacDonald and colleagues [7] investigated the ability to compensate for shifts of F1 and F2 in the unrounded vowel $/ \varepsilon /$ in toddlers (2-year-olds) and young children (4-year-olds) as compared to adults. The 2-year-olds did not show any response to the perturbation. On the other hand, the results for the 4-year-olds showed similar compensation with a larger token-to-token variability compared to the adults [7], replicating previous findings from Menard and colleagues [8, 9] who used a lip-tube to alter F1 and F2 of the rounded vowel /u/ produced by 4-year-old French speakers. These results indicate that while 4-year-olds are able to develop a compensatory strategy, they are unable to adapt and update and store their representations. Shiller, Gracco, \& Rvachew [13] manipulated the spectral properties of $/ \mathrm{s} /$ towards $/ \mathrm{J} /$ in a series of monosyllabic words in an auditory perturbation study involving 9 to 11 -year-old children. In this case, the results showed that the children were able to adapt to altered auditory feedback to a comparable degree as adults, albeit still with a larger token-to-token variability.

In the current study, we set out to further investigate to what extent children between $4-9$ years-old are able to use auditory feedback to compensate for and adapt to real-time shifts of F1 and F2 of the vowel /e:/ during the production of CVC words. 


\section{METHOD \& MATERIALS}

\subsection{Participants}

Two groups of Dutch speakers are involved in the experiment. Until now the first group consists of 15 children (8 female, 7 male; age range 4;1 - 8;7 y;m, mean $5 ; 8 \mathrm{y} ; \mathrm{m})$. The second group includes 37 adults (32 female, 5 male; age range 19 - 29 years, mean 22,4 years). None of the participants had current or previous speech or hearing problems.

\subsection{Stimuli}

The stimuli were three CVC words: /be:r/ (bear), /ve:r/ (feather), /pe:r/ (pear), all containing anclosemid front unrounded vowel. The Audapter software module [1] was used for auditory feedback perturbation. During perturbation conditions, the software recorded the speech signal, tracked and shifted the formant frequencies of the vowel, and played back the target word over headphones in real-time. The first formant was raised $25 \%$ and the second lowered $12.5 \%$, yielding a more open and more central vowel.

\subsection{Experimental procedure}

The participants were seated in front of a pcmonitor showing pictures of the three target words. A bird flying over one of the pictures cued the participant to speak the intended word, ensuring to mask the identity of the upcoming target word, thus limiting word preparation and selection possibilities by the speaker. The perturbation paradigm consisted of five phases (Figure 1): a practice phase where participants were made familiar with the paradigm and practiced the desired word length and loudness; a start phase which served as a baseline for unperturbed vowels; a ramp phase, where the perturbation was linearly ramped to the maximum; the hold phase where maximum perturbation was applied; and an end phase where the perturbation was suspended. The total number of tokens was 111. Due to fatigue and attention loss, children aged below 7;0 y;m participated in a shorter version of 75 tokens.

\subsection{Experiment debriefing}

Previous studies reported that adult participants were unable to notice perturbations when asked during debriefing $[1,4]$. In contrast, during the pilot phase of this study participants spontaneously indicated to have noticed manipulations of speech during direct feedback. To further investigate this matter, participants were asked during debriefing "Did you hear something odd when listening to your own voice?". The results of the debriefing responses were correlated with perturbation characteristics by means of a crosstab analysis.

Figure 1: Experimental paradigm. The number of trials for each phase was as follows (trial numbers of the short program are followed in parenthesis): Practice: 9 (9); Start: 27 (15); Ramp: 24 (18); Hold: 27 (18); End: 24 (15).

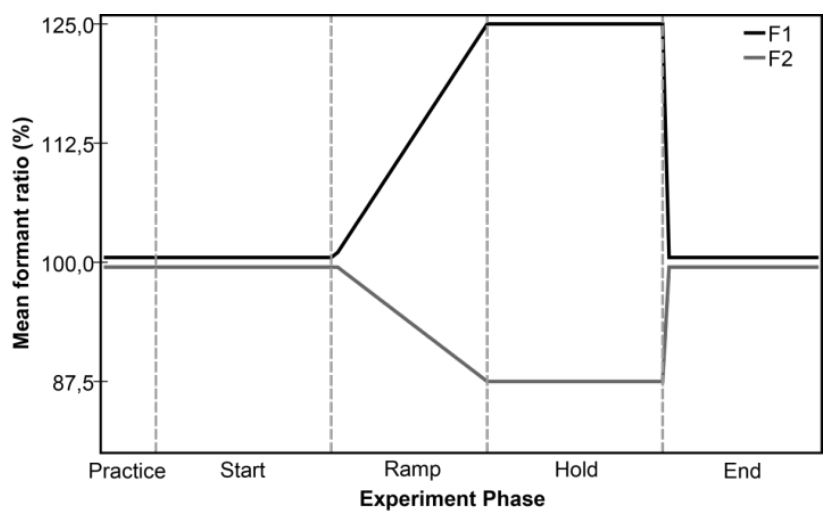

\subsection{Data analysis}

For each production, F1 and F2 were measured from steady-state portions of the produced vowels using custom PRAAT-scripts. The amount of compensation was quantified by calculating the difference in formant frequencies between the start and hold phase. This is a measure of motor learning: the ability to notice and act on the mismatch between the motor command and the corresponding auditory result. The amount of adaptation was quantified by calculating the differences in formant frequencies between the end and start phase. This is a measure of the after-effect of change in motor command, followed by recovery (de-adaptation).

\subsection{Statistical analyses}

Statistical analyses were carried out by means of Linear Mixed Model analyses separately for F1 and F2 data, with subject, phase, word, and repetition as correlated terms, and group and phase as fixed factors. The level of significance was set at $p<$ 0.05 . Significant main and interaction effects were further explored by means of univariate tests where appropriate or by a pairwise comparison using Fisher's Least Significant Difference Test.

\section{RESULTS}

\subsection{Normality and homoscedasticity}

Shapiro's test of normality and Levene's test of homoscedasticity were applied to the main outcome measures, prior to comparing the groups and phases 
by a series of statistical analyses. The results showed that both the requirements of normality and equality of variance were satisfied across all measures.

\subsection{Compensation and adaptation}

Figure 2 presents normalized F1 and F2 values by group for the start, ramp, stay and end phases.

Figure 2: Produced F1 and F2 frequencies, normalized to the mean values in the start phase.
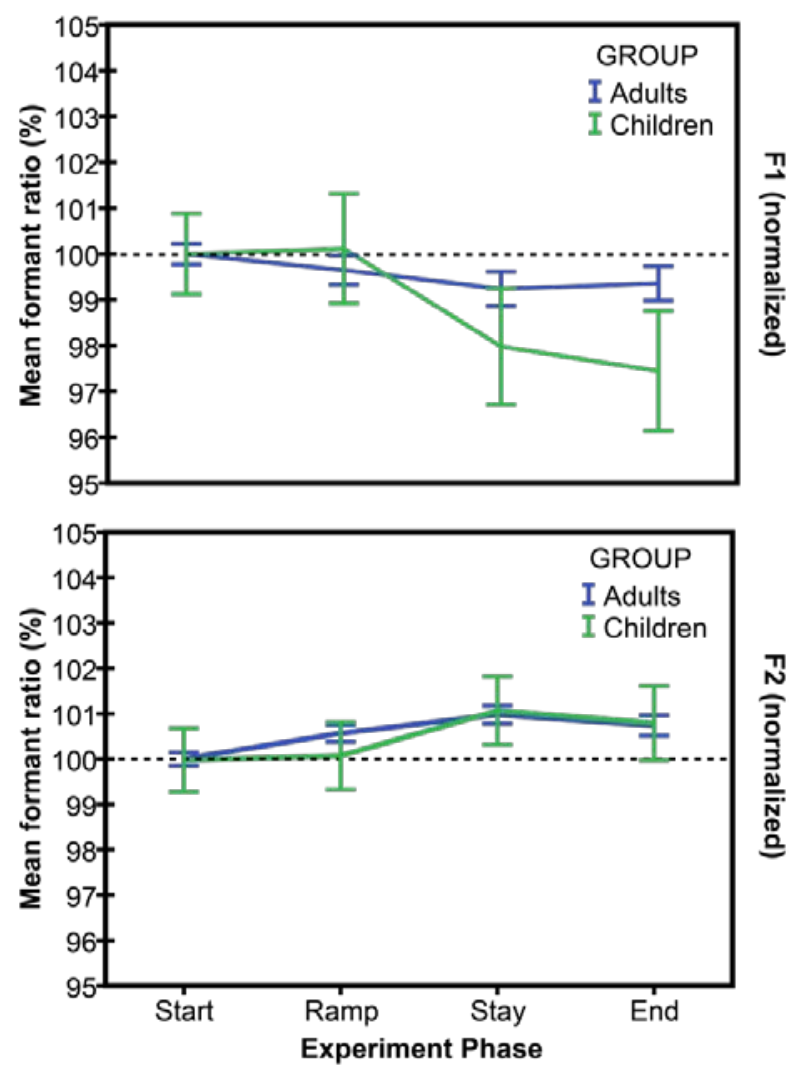

The results of the Linear Mixed Model analyses showed the following. With respect to the First Formant, a main effect of Group was found: $F(1,2121)=16.2, p<.001$, where on average children showed a higher response, as compared to the adults. A main effect of Phase was found: $F(2,2140)=4.0, p=.018$. Across groups, responses in the Stay and End phases were significantly larger when compared to the Start and Ramp phases. A Group $x$ Phase interaction effect $[F(2,2140)=4.0$, $p=.018]$ showed that for some of the phases, children showed a higher response compared to adults. To further investigate these results, a series of post-hoc analyses were carried out. Separately for each group, it was found that adults showed compensation $(p=.005)$, but an adaptation effect was absent $(p=.093)$. In contrast, children showed compensation and adaptation (both $p<.001$ ). When comparing the groups for each phase individually, it was found that children showed a stronger compensation response in the Stay phase ( $p=.004)$; and a stronger adaptation response in the End phase $(p=.003)$.

The statistical results for the Second Formant failed to display a main effect of Group: $F(1,2132)$ $=2.0, p=.155$, when compared across all phases. A main effect of Phase was present: $F(2,2147)=18.3$, $p<.001$, showing that responses in the Stay and End phases were significantly larger when compared to the Start and Ramp phases. An insignificant Group x Phase effect: $F(2,2147)=$ $.209, p=.811$, showed that across phases, both groups responded equally. Post-hoc analyses indicated that adults showed significant compensation and adaptation (both $p<.001$ ), while children showed a significant compensation effect ( $p=.003)$, but no adaptation $(p=.063)$. When comparing groups for each phase separately, it was found that there were no group differences in the Stay phase: $p=.354$ or End phase: $p=.786$.

\subsection{Correlations with age in the group of children}

To see whether it was possible to detect developmental changes in the group of children, compensation and adaptation responses were linearly correlated with age. The results of the correlation analyses (see Figure 3) showed that there were no noticeable effects of age on compensation or adaptation response.

Figure 3: Scatter plots of age and compensation response (top) and adaptation response (bottom) for the group of children.
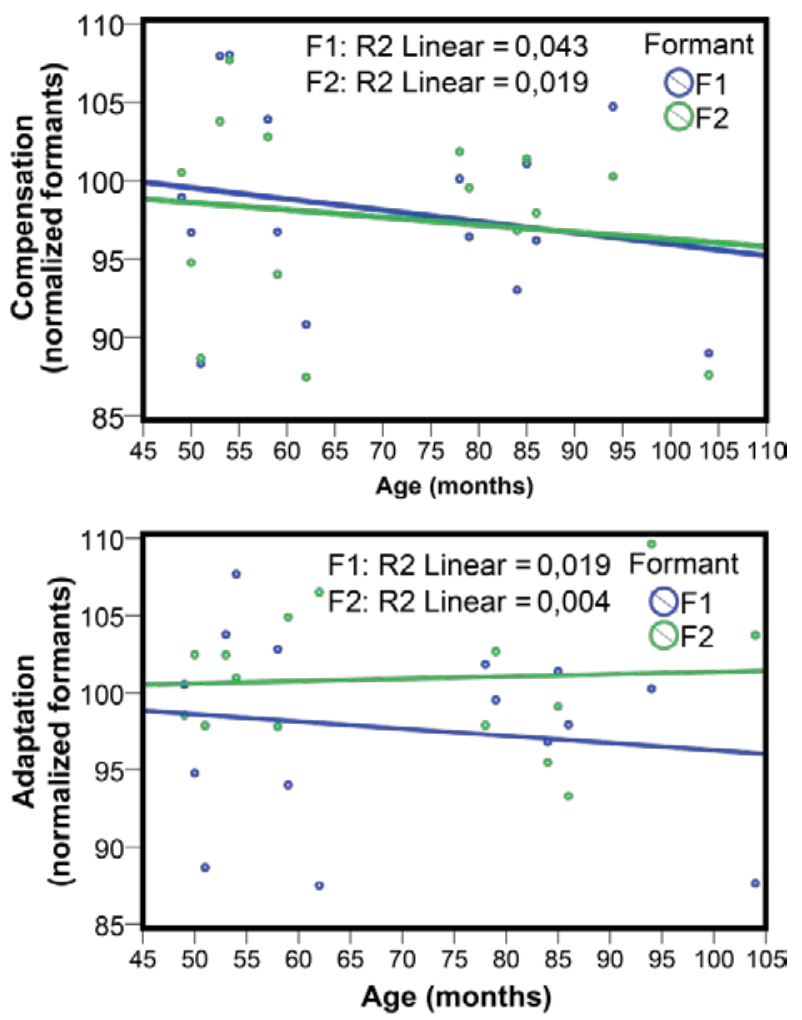


\subsection{Experiment debriefing}

The results of the experiment debriefing (see Figure 4) showed that around $65 \%$ of the adult participants indicated to have noticed manipulations of the stimuli, while some of them reported having consciously undertaken action. The crosstab analysis showed no correlation between debriefing response and perturbation of F1 and F2.

Figure 4: Adult participants' responses to the debriefing question "Did you hear something odd when listening to your own voice?".

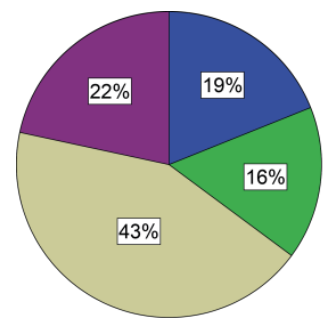

$\nabla$ No

$\nabla$ No, but recalled possible changes after pointing out vowel manipulations

Yes, noticed manipulations during experiment

$\bowtie$ Yes, noticed manipulations, and acted on it during experiment

\section{DISCUSSION}

\subsection{Summary of findings}

In the present study, we investigated auditory perturbation of F1 and F2 of the vowel /e:/ in CVCwords in 4 to 9 year-old children compared to young adults (aged 19 - 29 years). The results showed that the children were able to compensate and adapt in a similar or larger degree compared to the young adults, even though the proportion of speakers displaying a consistent compensatory response was higher in the group of adults. Furthermore, results did not show differences in token-to-token variability across groups, in contrast to previous reports $[7-9,13]$.

\subsection{Compensation and adaptation across groups}

The stronger response in compensation for F1 and F2 in the group of children suggest that auditorymotor properties are less ingrained as compared to adult speakers. Furthermore, the presence of adaptation effects in the group of children for the first formant suggest that the length of the ramp and stay phases were adequate, that is, there were enough trials to induce short-term training and learning, even during the shorter program designed for some of the children. The stronger and longer adaptation response for the first formant in the group of children suggests that older adults revert faster to the ingrained original representation of the speech sound. The absence of an adaptation response in the group of children for the second formant is possibly due to a relatively small group size, in combination with a large within-group variance.

It cannot be ruled out that the stronger adaptation and compensation effects in children as compared to the adult speakers might be due to a relative larger formant vowel space in which they operate, as has been noticed in $[6,15]$. Research into proportionalities of vowel space across participant groups will be needed to establish this factor.

\subsection{Developmental effects}

The results did not show a linear correlation of age with compensation and adaptation responses in the group of children. The absence of such age-related effects could indicate that learning strategies of auditory-motor integration do not change significantly in the age span of 4-9 years. However, it might also be due to a small a number of observations or due to large within-group differences. These within-group differences might result from different strategies that were employed with respect to focussing on somatosensory feedback versus focussing on auditory feedback [5]. Future studies should include a larger number of children, and the investigation of potential tradeoffs between different types of feedback are warranted.

\subsection{Effects of noticing stimuli manipulations}

The results showed that overtly noticing stimuli manipulations do not entail different adaptation and compensation strategies for F1 and F2 in adult speakers, even if they said to have applied an overt response to battle or compensate for the stimuli manipulations. This indicates that subconscious compensation and adaptation during this perturbation experiment was strong and sustainable.

\section{ACKNOWLEDGEMENTS}

This study was funded by the Netherlands Organization for Scientific Research (NWO-VENI grant 275-89-016 awarded to the first author). The authors gratefully thank the participants, especially the children and their parents or caretakers for their time and effort, Anniek van Doornik-van der Zee and Margoke Nijssen for their help with participant recruitment, and Shanqing Cai for his help with the Audapter software.

\section{REFERENCES}

[1] Cai, S., et al. 2010. Adaptive auditory feedback control of the production of formant trajectories in the Mandarin triphthong /iau/ and its pattern 
of generalization, The Journal of the Acoustical Society of America, 128, 2033-2048.

[2] Guenther, F.H., Hampson, M., Johnson, D. 1998. A theoretical investigation of reference frames for the planning of speech movements, Psychological Review, 105, 611-33.

[3] Guenther, F.H., Perkell, J.S., 2004. A neural model of speech production and its application to studies of the role of auditory feedback in speech In: Speech Motor Control in Normal and Disordered Speech, B. Maassen, et al., Eds. Oxford, UK: Oxford University Press, 29-50.

[4] Houde, J.F., Jordan, M.I. 2002. Sensorimotor Adaptation of Speech I: Compensation and Adaptation, Journal of Speech, Language, and Hearing Research, 45, 295-310.

[5] Katseff, S., Houde, J., Johnson, K. 2012. Partial Compensation for Altered Auditory Feedback: A Tradeoff with Somatosensory Feedback?, Language and Speech, 55, 295-308.

[6] Lee, S., Potamianos, A., Narayanan, S. 1999. Acoustics of children's speech: Developmental changes of temporal and spectral parameters, The Journal of the Acoustical Society of America, 105, 1455.

[7] MacDonald, E.N., et al. 2012. Children's Development of Self-Regulation in Speech Production, Current Biology, 22, 113-117.

[8] Ménard, L., Perrier, P., Aubin, J. 2013. The role of auditory feedback in speech development: A study of compensation strategies for a lip-tube perturbation, Proceedings of Meetings on Acoustics, 19, 060181.
[9] Ménard, L., et al. 2008. Compensation strategies for a lip-tube perturbation of French [u]: An acoustic and perceptual study of 4-yearold children, The Journal of the Acoustical Society of America, 124, t1192-1206.

[10] Perkell, J. 2012. Movement goals and feedback and feedforward control mechanisms in speech production, Journal of Neurolinguistics, 25, 382-407.

[11] Perkell, J., et al. 2007. Effects of masking noise on vowel and sibilant contrasts in normalhearing speakers and postlingually deafened cochlear implant users, The Journal of the Acoustical Society of America, 121, 505-18.

[12] Perkell, J., et al. 1997. Speech motor control: Acoustic goals, saturation effects, auditory feedback and internal models, Speech Communication, 22, 227-250.

[13] Shiller, D., Gracco, V., Rvachew, S. 2010. Auditory-Motor Learning during Speech Production in 9-11-Year-Old Children, PLoS ONE, 5, e12975.

[14] Villacorta, V.M., Perkell, J.S., Guenther, F.H. 2007. Sensorimotor adaptation to feedback perturbations of vowel acoustics and its relation to perception, The Journal of the Acoustical Society of America, 122, 2306-19.

[15] Vorperian, H.K., Kent, R.D. 2007. Vowel acoustic space development in children: A synthesis of acoustic and anatomic data, Journal of Speech, Language, and Hearing Research, 50, 1510-1545. 